\title{
Influence of Aerobic Exercise on Sleep and Salivary Melatonin in Men
}

\author{
Kaylee M Pobocik, PT, DPT ${ }^{1}$, Seth A Von Rentzell ${ }^{1}$, Abigail J Leonard ${ }^{1}$, Amber N Daye $^{2}$ and \\ Elizabeth S Evans, $P h D^{1^{*}}$
}

${ }^{1}$ Department of Physical Therapy Education, Elon University, USA

${ }^{2}$ Department of Mathematics and Statistics, Elon University, USA

*Corresponding author: Elizabeth S. Evans, PhD, Department of Physical Therapy Education, 2085 Campus Box, Elon University, Elon, NC 27244, USA, Tel: (336)-278-63534, Fax: (336)-278-4189

\begin{abstract}
Background: Insomnia is a sleep disorder that affects approximately 50-70 million US adults. Previous studies have examined the impact of exercise as a non-pharmacologic mechanism for improving sleep and reducing insomnia. The body's melatonin secretion is considered an internal sleep regulator (as opposed to external sleep regulators such as medications) and may be improved by regular exercise. Therefore, the purpose of this study was to evaluate the effect of an 8-week aerobic exercise intervention on nighttime salivary melatonin concentrations and self-reported sleep quality in a cohort of adult men.
\end{abstract}

Methods: Utilizing an experimental pre-test post-test design, 17 previously sedentary men ages 18-44 years completed 8 weeks of moderate-intensity aerobic exercise. Sleep Quality and was assessed pre-intervention and post-intervention using the Pittsburgh Sleep Quality Index (PSQI). Salivary melatonin concentrations were assessed pre-intervention, mid-intervention, and post-intervention at 20:00h, 22:00h, and 03:00h using Enzyme-Linked Immunosorbent Assay (ELISA) techniques. Paired sample t-tests were used to compare changes in PSQI scores from pre-intervention to post-intervention, as well as to compare salivary concentrations at 20:00h, 22:00h, and 03:00h during the pre-intervention, mid-intervention, and post-intervention collections.

Results: PSQI scores significantly decreased from pre-intervention to post-intervention, indicating significant improvement in sleep quality $(p=0.046)$. Salivary melatonin concentrations increased significantly from pre-intervention to post-intervention at $20: 00 \mathrm{~h}$, as well as from $20: 00 \mathrm{~h}$ to $3: 00 \mathrm{~h}$ within the post-intervention collection period $(\mathrm{p}=$ 0.015-0.037).
Conclusions: Moderate aerobic exercise may increase the production of nighttime melatonin, thus improving internal sleep regulation and positively impacting sleep quality in previously sedentary adult men. Individuals with sleep problems may consider using exercise to improve aspects of sleep.

\section{Keywords}

Insomnia, Circadian rhythm, Melatonin, Physical activity

\section{Introduction}

Insomnia is a sleep disorder that affects approximately 50-70 million US adults [1]. Affected individuals can experience cognitive problems, decreased performance and productivity, and reduced quality of life [16]. Additionally, insomnia can be associated with multiple adverse health conditions including hypertension, coronary heart disease, diabetes, cancer, depression, and obesity [3-6]. Adverse effects of sleep disorders on health and wellness can also have a negative economic impact, leading to increased healthcare costs and financial burden in the workplace $[3,7]$.

The hormone melatonin is synthesized by the pineal gland in the brain, and its secretion is linked to the dark cycle of the body's 24-hour circadian rhythm [8-12]. Melatonin's production is blunted during the daytime (e.g. the light cycle); and at nighttime, norepinephrine stimulates the pineal gland to synthesize melatonin from its precursor's serotonin and tryptophan, thus

Citation: Pobocik KM, Rentzell SAV, Leonard AJ, Daye AN, Evans ES (2020) Influence of Aerobic Exercise on Sleep and Salivary Melatonin in Men. Int J Sports Exerc Med 6:161. doi.org/10.23937/24695718/1510161

Accepted: April 14, 2020; Published: April 16, 2020

Copyright: (C) 2020 Pobocik KM, et al. This is an open-access article distributed under the terms of the Creative Commons Attribution License, which permits unrestricted use, distribution, and reproduction in any medium, provided the original author and source are credited. 
inducing sleepiness [8-10]. Typically, melatonin levels begin to increase at the onset of darkness, peak between 3:00-4:00 am (e.g. 03:00-4:00h), and then slowly descend [8]. Therefore, this increase and decrease in melatonin levels occurring at the respective onset and end of the nighttime enable the body to distinguish between the light and dark cycles of the circadian rhythm [8]. Since melatonin is an endogenously produced substance that affects the body's circadian rhythm (as opposed to an ingested medication), melatonin is considered an internal sleep facilitator [8].

Previous studies have examined the impact of regular physical activity and exercise as a non-pharmacologic mechanism for improving sleep and reducing insomnia across the lifespan in individuals with and without sleep impairments $[1,2,4,6,13,14]$. Collectively, these investigations have shown that habitual physical activity and exercise are associated with positive outcomes such as improved sleep quality, reduced sleep latency, fewer nighttime awakenings, and overall reduced insomnia, along with increased salivary melatonin responses $[2,6,13,15]$. Thus, regular physical activity and exercise may be a potentially beneficial, safe, and cost-effective approach for manipulating the body's circadian rhythm and promoting improved sleep $[6,15]$.

However, definitive correlations between exercise time of day (e.g. exercise performed in the morning vs. the afternoon vs. the evening) and their respective effects on the circadian rhythm, sleep, and melatonin levels at specific points during the day and night are not well-established $[9,16,17]$. While previous studies have examined the effects of exercise on salivary melatonin levels during specific times in the morning and afternoon, further assessment of the effects of exercise on salivary melatonin levels throughout the night is crucial for developing an increased understanding of the relationship between melatonin and the sleep-wake cycle [11]. Therefore, the purpose of this study was to evaluate the effect of an 8-week aerobic exercise intervention on nighttime salivary melatonin levels and self-reported sleep quality in adult men, hypothesizing that salivary melatonin concentrations and self-reported sleep quality would both increase pre- to post-intervention.

\section{Methods}

\section{Participants}

Individuals were eligible to participate if they were men, between the ages of 18-44 years, and had been exercising for less than 30 minutes per day, 3 days per week, for at least 1 year prior to study enrollment. Individuals were excluded if they were regularly taking a prescribed medication for a sleeping disorder or had a cardiopulmonary, renal, metabolic, or musculoskeletal disorder that could be exacerbated by exercise, or rendered exercise unsafe. Participants were recruited through university faculty, staff, and student email listservs, as well through informational fliers posted around the university and surrounding geographical locations. All aspects of the study were approved by the Elon University institutional review board in the spirit of the Helsinki Declaration prior to beginning recruitment of participants. All participants provided written informed consent after being informed of study procedures, risks, and benefits, and all participant rights were protected throughout the duration of the study. After all recruitment efforts were exhausted, this study included 17 participants. This sample size is comparable to the sample sizes of other studies with similar designs and outcomes $[2,15,18-26]$.

\section{Overview of the study design}

This study employed a single-group experimental pre-test post-test design. Each participant completed an 8-week aerobic exercise intervention, where they were required to exercise for 3-4 sessions per week at a moderate intensity (e.g. 50-70\% of $\mathrm{VO}_{2 \text { peak }}$ ) for $30-45$ minutes per session. Before beginning the intervention, participants completed a baseline testing session, which included assessment of physical characteristics (e.g., age, height, body mass, body mass index, and percent body fat), peak aerobic capacity $\left(\mathrm{VO}_{\text {2peak }}\right)$, and administration of a sleep quality questionnaire (e.g., the Pittsburgh Sleep Quality Index). Upon completion of the intervention, participants returned for a follow-up testing session, which was identical to the baseline testing session. Saliva samples were collected in the late evening and early morning hours at 3 time points throughout the study (e.g. baseline, the mid-point of the intervention, and upon completion of the intervention) in order to examine salivary melatonin responses across time. Participant enrollment and participation occurred over a period of approximately 18 months, with the first participant enrolling and performing their baseline testing session in June 2017, and the last participant completing their exercise intervention and follow-up testing session in December, 2018. All baseline and follow-up testing sessions occurred in the university's School of Health Sciences Human Performance Laboratory, while the aerobic exercise intervention sessions occurred at participant-chosen locations. In order to reduce the potential effects of bias and confounders, all participants were instructed to follow the same set of pre-testing guidelines prior to their baseline testing session, post-intervention testing session, and saliva collections (details described in the following sections). All participants were also given the same instructions, guidance, and demonstrations on how to complete the sleep quality questionnaire and testing procedures during the baseline and post-intervention testing sessions, as well as how to record and document the details of each session of their 8-week aerobic exercise intervention, and how to properly collection and store their saliva samples (details described in the following sections). All equipment 
used to collect data during the baseline and post-intervention testing sessions was the same for each participant, and all equipment was properly calibrated prior to each testing session.

\section{Baseline testing session}

Prior to the baseline testing session, participants were instructed to refrain from eating for 2 hours, as well as to refrain from exercise, caffeine, alcohol, and anti-inflammatory medications for 24 hours. Participants were instructed to drink water as they normally would during the 24 hours prior to testing. Compliance with pre-testing instructions was confirmed with each participant at the beginning of the testing session.

After providing written informed consent, participants completed a medical history questionnaire along with the Physical Activity Readiness Questionnaire (PAR-Q) to verify that each individual met the inclusion criteria for the study [19]. Participant self-reported sleep quality was assessed using the Pittsburgh Sleep Quality Index (PSQI), an instrument with a high validity and test-retest reliability for patients with sleep disturbances, including insomnia [20]. Height and body mass were measured using a portable stadiometer and balance beam scale (Health O Meter ${ }^{\circledast}$, McCook, IL). Percent body fat was measured using tetrapolar Bioelectrical Impedance Analysis (Biodynamics Corp., Shoreline, WA). Resting heart rate was measured using a Polar telemetry system (Polar Electro Inc., Bethpage, NY) and resting blood pressure was measured using a stethoscope and sphygmomanometer (Omron Healthcare Inc., Lake Forest, IL).

Peak aerobic capacity $\left(\mathrm{VO}_{2 \text { peak }}\right)$ was assessed using a Modified Bruce Treadmill Protocol [21]. Expired gases were collected using a 2400 True One Metabolic Measurement System (Parvo Medics, Salt Lake City, UT). Resting metabolic data was collected for 3 minutes prior to beginning the first stage of the test. During the first stage of the test, participants walked on the treadmill at $1.7 \mathrm{mph}$ and $0 \%$ grade for 3 minutes. Each subsequent test stage also lasted for 3 minutes, during which treadmill speed and grade increased until volitional fatigue. Expired gases were collected continuously throughout the test, while heart rate and Rating of Perceived Exertion (RPE) were recorded at the end of each min- ute. The highest $\mathrm{VO}_{2}$ measured by the metabolic system during the last stage of the test was recorded at the participant's $\mathrm{VO}_{2 \text { peak }}$. Once the $\mathrm{VO}_{2 \text { peak }}$ test was complete, participants entered an active cool-down where they were allowed to walk on the treadmill at a self-selected pace. Heart rate and blood pressure were monitored until they had returned to near-resting levels.

\section{Aerobic exercise intervention}

The results of the baseline $\mathrm{VO}_{2 \text { peak }}$ test were used to compile an 8-week moderate-intensity aerobic exercise intervention specific to each participant, according to American College of Sports Medicine guidelines [23]. Heart rates corresponding to $50 \%, 55 \%, 60 \%, 65 \%$, and $70 \% \mathrm{VO}_{2 \text { peak }}$ were calculated by regressing average $\mathrm{VO}_{2}$ values for each minute of the $\mathrm{VO}_{2 \text { peak }}$ test against the minute-by-minute heart rate responses. Participants were instructed to exercise for 30-45 minutes per day, 3-4 days per week, using the target heart rates that corresponded to $50-70 \%$ of $\mathrm{VO}_{2 \text { peak }}$. A general overview of the week-by-week progression of the exercise intervention is shown in Table 1. Participants were given a Pyle Sports ${ }^{\circledR}$ PHRM34 heart rate monitor and watch (Pyle Audio, Inc., Brooklyn, NY) for them to ensure that they were exercising within the prescribed target heart rate range during each exercise session. Participants were allowed to self-select their preferred modes of exercise, and researchers reviewed types of aerobic exercise modes with each participant, along with proper warmup and cool-down techniques. Participants recorded details of each exercise session (i.e. exercise mode, heart rates, and exercise duration) using the My Fitness Pal App (Under Armour, Inc., Baltimore, MD).

\section{Post-intervention testing session}

Within two weeks of completing the aerobic exercise intervention, participants returned for post-intervention testing, which occurred in an identical manner to the baseline testing session. Participants were instructed to follow the same pre-testing guidelines prior to the post-intervention testing session as they did for the pre-intervention testing session, and compliance was again confirmed with each participant at the beginning of the testing session. Height, body mass, percent body fat, and resting vitals were measured and recorded as

Table 1: Aerobic Exercise Prescription for the 8-week Intervention Period.

\begin{tabular}{|l|l|l|l|}
\hline Week & Exercise Intensity $\left(\% \mathbf{~ V O}_{\text {2peak }}\right)$ & Exercise Duration (minutes) & Exercise Frequency (days/week) \\
\hline 1 & $50-55$ & $30-35$ & 3 \\
\hline 2 & $50-55$ & $30-35$ & $3-4$ \\
\hline 3 & $50-55$ & $35-40$ & $3-4$ \\
\hline 4 & $55-60$ & $35-40$ & $3-4$ \\
\hline 5 & $55-60$ & $35-40$ & $3-4$ \\
\hline 6 & $60-65$ & $35-40$ & $3-4$ \\
\hline 7 & $60-65$ & $40-45$ & $3-4$ \\
\hline 8 & $65-70$ & $40-35$ & $3-4$ \\
\hline
\end{tabular}


previously described. Sleep quality was once again assessed using the PSQI, and $\mathrm{VO}_{2 \text { peak }}$ was assessed using the Modified Bruce Treadmill Protocol as previously described [21].

\section{Collection of saliva and determination of salivary melatonin concentrations}

Participants were given materials and instructions for performing their own saliva collections. Saliva was collected via a passive drool technique into sterile $1.5 \mathrm{ml}$ polypropylene conical tubes. Participants were instructed to drool into the tubes over a 4-minute period and place their saliva specimens in their home freezer until study completion. Participants performed saliva collections at baseline, during Week 4 of the intervention (e.g. the intervention mid-point), and upon completion of post-intervention testing session (e.g. post-intervention).For the baseline saliva collection, participants collected saliva at 8:00 pm (e.g. 20:00h) and 10:00 pm (e.g. 22:00h) on the day following the baseline testing session and at 3:00 am (e.g. 03:00h) on the following morning. For the mid-point saliva collection, participants chose a day during Week 4 of the intervention to perform the collection, again collecting samples at 20:00h, 22:00h, and $3: 00 \mathrm{~h}$. For the post-intervention saliva collection, participants collected saliva at 20:00h and 22:00h on the day following the post-intervention testing session and at 3:00h on the following morning. Prior to collecting saliva samples, participants were instructed to follow the same pre-testing guidelines that they did before the baseline and post-intervention testing sessions (e.g., to refrain from eating for 2 hours, as well as to refrain from exercise, caffeine, alcohol, and anti-inflammatory medications for 24 hours). All participant saliva samples were stored in a $-20^{\circ} \mathrm{C}$ freezer until time of analysis.

Saliva samples were centrifuged at $4{ }^{\circ} \mathrm{C}$ for $15 \mathrm{~min}$ utes at $3500 \mathrm{rpm}$ using a Sorvall ST $16 \mathrm{R}$ refrigerated centrifuge (Thermo Fisher Scientific, Inc., Waltham, MA). Salivary melatonin concentrations were measured using enzyme-linked immunosorbent assay (ELISA) kits (Salimetrics, State College, PA). The salivary melatonin ELISA had an analytical sensitivity of $1.37 \mathrm{pg} / \mathrm{mL}$, mean intra-assay coefficients of variation of $3.9-7.4 \%$, and mean inter-assay coefficients of variation of $4.6-15.6 \%$. All samples were run in duplicate.

\section{Statistical analysis}

Statistical analyses were performed using SAS Version 9.4 TS1M6. Paired-sample t-tests were used to compare changes in physical characteristics (e.g., body mass, BMI, percent body fat, and $\mathrm{VO}_{2 \text { peak }}$ ) and PSQI scores from pre-intervention to post-intervention. Paired sample t-tests were also used to compare salivary melatonin concentrations at 20:00h, 22:00h, and 03:00h within and between the pre-intervention, mid-intervention, and post-intervention collection periods. Each variable was centered at the mean so that true mean differences could be compared. Significance was set a priori at $p<$ 0.05. All data are displayed as mean \pm Standard Deviation (SD).

\section{Results}

\section{Participant physical characteristics and pittsburgh sleep quality index scores}

Seventeen individuals participated in this study. Participant physical characteristics and sleep quality scores are presented in Table 2. There were no significant changes for any physical characteristic (e.g. body mass, $\mathrm{BMI}$, and percent body fat) or $\mathrm{VO}_{2 \text { peak }}$ from pre-intervention to post-intervention ( $p=0.219-0.573$ ). Sleep quality scores, as assessed by the PSQI, significantly decreased from pre-intervention to post-intervention, indicating a significant improvement in sleep quality $(p=0.046)$.

\section{Adherence to the exercise intervention}

Participants' exercise mode, time, and average heart rate during each exercise session were tracked via the My Fitness Pal App (Under Armour, Inc., Baltimore, MD). Any aerobic activity was acceptable, as long it involved continuous muscular activity at the intensity and duration range prescribed for that session. Exercise modes utilized by participants included walking, biking, kayaking, stationary cardio machines, running, and hiking. Participants were also instructed how to properly warm up and cool down before and after each exercise session and were advised to

Table 2: Physical Characteristics, $\mathrm{VO}_{2 \text { peak }}$, and Pittsburgh Sleep Quality Index Scores $(\mathrm{n}=17)$.

\begin{tabular}{|c|c|c|c|c|}
\hline \multirow[t]{2}{*}{ Parameter } & \multicolumn{2}{|c|}{ Pre-Intervention } & \multicolumn{2}{|c|}{ Post-Intervention } \\
\hline & Mean \pm SD & $95 \% \mathrm{Cl}$ & Mean \pm SD & $95 \% \mathrm{Cl}$ \\
\hline Age (years) & $32 \pm 6$ & 29-35 & -- & -- \\
\hline Height (cm) & $175.7 \pm 9.1$ & 171.1-180.4 & -- & -- \\
\hline Body Mass (kg) & $96.9 \pm 22.0$ & $85.6-108.2$ & $96.6 \pm 21.7$ & $85.4-107.8$ \\
\hline $\operatorname{BMI}\left(\mathrm{kg} / \mathrm{m}^{2}\right)$ & $31.2 \pm 5.5$ & $28.4-34.1$ & $31.1 \pm 5.7$ & $28.2-34.1$ \\
\hline Body Fat (\%) & $26.7 \pm 7.0$ & 23.1-30.3 & $26.7 \pm 7.1$ & $22.7-30.0$ \\
\hline $\mathrm{VO}_{2 \text { peak }}(\mathrm{mL} / \mathrm{kg} / \mathrm{min})$ & $33.8 \pm 7.7$ & $29.9-37.8$ & $34.6 \pm 8.6$ & $30.2-39.0$ \\
\hline PSQI Score* & $6 \pm 2$ & $5-7$ & $4 \pm 2$ & $3-6$ \\
\hline
\end{tabular}

BMI: Body Mass Index, PSQI: Pittsburgh Sleep Quality Index, SD: Standard Deviation, CI: Confidence Interval $\mathrm{p}=0.046$. 


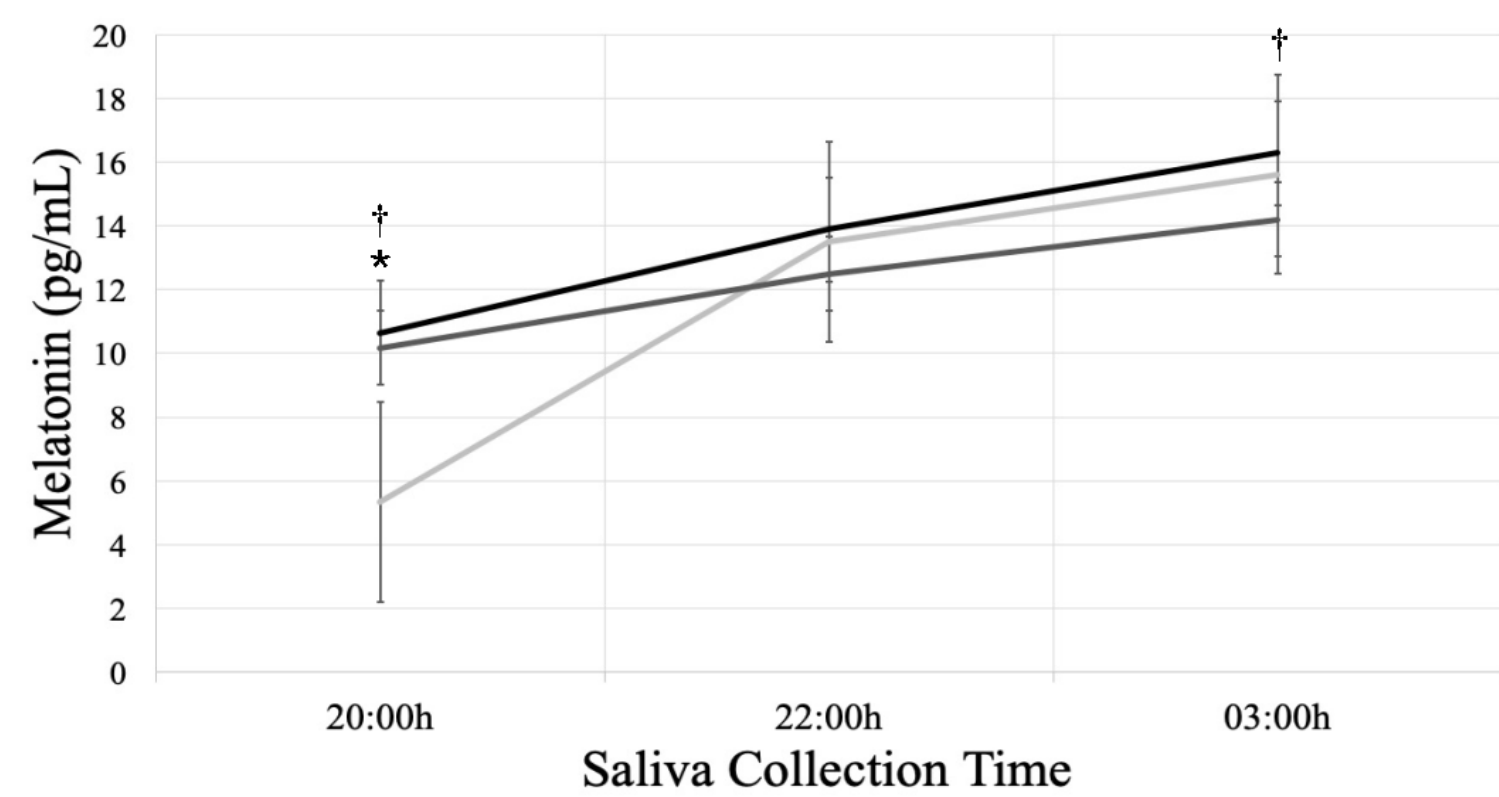

\section{-Pre -Week 4 -Post}

Figure 1: Salivary Melatonin Concentrations at Pre-Intervention, Mid-Intervention, and Post-Intervention.

${ }^{*} p=0.015$ for comparing 20:00h at pre-intervention vs. post-intervention; ${ }^{\dagger} p=0.037$ for comparing 20:00h and 03:00h at post-intervention.

do each for approximately 5 minutes. Approximately $88 \%$ of all prescribed weekly exercise sessions were completed. Over the course of the 8-week intervention, approximately $4 \%$ of the exercise sessions exceeded the prescribed exercise duration. Participants reported that they stayed within $10 \mathrm{bpm}$ of the prescribed target heart rate range for workouts.

\section{Salivary melatonin concentrations}

Salivary melatonin concentrations at each time point are presented in Figure 1. Salivary melatonin concentrations increased significantly from pre-intervention to post-intervention at the 20 :00h collection time point ( $p$ $=0.015$ ). Additionally, salivary melatonin concentration increased significantly from 20:00h to 03:00h within the post-intervention collection period ( $p=0.037$ ). No significant differences were observed for any other pairwise comparison of salivary melatonin concentration ( $p$ $=0.188-0.951$ ).

\section{Discussion}

The purpose of this study was to evaluate the effect of an 8-week moderate intensity aerobic exercise intervention on nighttime salivary melatonin levels and self-reported sleep quality in adult men who were previously sedentary. There was a significant increase in salivary melatonin concentration at 20:00h from pre-intervention to post-intervention, as well as a significant rise in salivary melatonin concentration from 20:00h to 03:00h post-intervention. Additionally, PSQI scores significantly decreased from pre-intervention to post-intervention. No significant changes in body mass, BMI, percent body fat, or peak aerobic fitness were observed from baseline to post-intervention. The absence of sig- nificant improvements in body composition and peak aerobic fitness were not surprising, as participants were not instructed to alter their nutritional practices (other than following the pre-testing guidelines described previously), and the moderate exercise volume of the intervention may not have provided enough of a physiological stimulus to significantly increase $\mathrm{VO}_{2 \text { peak }}$.

The results of this study suggest that the aerobic exercise intervention may have improved the ability to fall asleep by increasing melatonin production at 20:00h. Evidence of these changes in melatonin production may be reflected in the significant improvements in self-reported sleep quality, as depicted by decreasing the average post-intervention PQSI score to less than 5 (e.g. the cutoff point for differentiating poor vs. good sleep as measured by the PSQI) [20].

With regard to previous studies examining the effects of exercise on sleep outcomes, Inoue, et al. discovered that high frequency physical activity reduced the incidence of insomnia in older adults [13]. Chen, et al. studied the effects of an 8-week aquatic exercise program on adults with sleep impairments and found exercise to be associated with improved sleep quality, reduced sleep latency, and fewer awakenings [6]. Kashefi, et al. also identified that 8 weeks of aerobic exercise significantly improved sleep quality in middle-aged women suffering from insomnia [2]. Furthermore, Yang, et al. found that participation in exercise training programs of 10-16 weeks that included aerobic and resistive training had moderately positive effects on sleep quality in adults over the age of 40 years [14]. Thus, the results of this study support the utilization of exercise as an approach to potentially 
improve onset of sleep and sleep quality.

The melatonin findings of this study are also supported by previous literature. Lee, et al. showed increased melatonin concentrations in sedentary adult men following 6 weeks of sun exposure for $30 \mathrm{~min}$ utes per day, along with 30 minutes of moderate intensity aerobic exercise 5 times per week [25]. Cai, et al. found that 10 weeks of moderate to high intensity step aerobics training for 40-45 minutes per day, 3 times per week increased melatonin concentration and improved PSQI scores in post-menopausal women [26]. Buxton, et al. found that moderate-high intensity daytime exercise can elicit an acute melatonin response in healthy adult men, suggesting that appropriately timed exercise can manipulate the circadian rhythm in a non-pharmacological way [18]. In contrast however, a few studies provide conflicting evidence with regard to sleep, exercise, and the effect on melatonin levels $[9,17]$. Carr, et al. and Theron, et al. found that daytime exercise increased plasma melatonin concentration acutely with concentrations falling back to normal post-exercise, whereas Montelone, et al. found that evening exercise led to significantly lower melatonin levels 3 hours after exercise [17,27-29]. Atkinson, et al. suggests that varying effects of exercise on melatonin levels such as this may be related to participant age, gender, individual activity level or exercise volume, and form of exercise intervention [17]. Additionally, variations in findings may be attributed to differences in lighting conditions and the time of day at which the study subjects exercised $[8,9,12,15]$.

The mechanisms underlying increased melatonin concentrations and improved sleep outcomes may relate to the effect of melatonin on circadian changes in core body temperature [9]. In particular, melatonin promotes a decrease in core body temperature with metabolic heat loss and a decrease in heat production, potentially allowing for a faster sleep onset $[10,15]$. Regular exercise has been shown to improve sleep, potentially due to its effects on core temperature and heat loss $[8,15,24]$. Exercise may also modulate the relationships that exist between melatonin, norepinephrine, and epinephrine, thus promoting increases in melatonin synthesis and secretion $[11,17]$.

A major strength of this study is that it is one of few to examine serial changes in salivary melatonin concentrations along with self-reported sleep quality in response to an exercise training intervention. The salivary collection times were chosen to encompass the linear rise in melatonin levels beginning at dusk and peaking at 03:00h-04:00h. Participants were physically inactive prior to enrollment so that the effect of the 8-week exercise intervention would be more clearly observed. As with any study, there are limitations to this current investigation. This study employed single group design in which participant characteristics were homogenous and all participants underwent the same type of exercise intervention. Therefore, there was no concurrent control group, or group with differing characteristics (e.g. women, individuals younger than 18 years or older than 44 years, athletes, etc.) with which to compare results. Participant enrollment occurred during all four seasons of the calendar year, during which the lengths of day and night are variable. Participants were allowed to maintain their normal routine outside of the exercise intervention, and factors such as timing of food and drink consumption, light exposure, bedtime, waking time, and other sleep hygiene specifics were not controlled for, other than the pre-assessment guidelines that each participant followed prior to baseline and post-intervention testing, and before each salivary collection. This study also did not control for mode of exercise, exercise time of day, or exercise setting (e.g. indoors vs. outdoors, exercising alone vs. exercising with others). Study investigators were not physically present participant exercise sessions, and thus relied on participant documentation of the details of their exercise sessions through My Fitness Pal (Under Armour, Inc., Baltimore, $\mathrm{MD})$. However, participants were encouraged to reach out to study investigators with questions or further guidance throughout the duration of the study. Other potential underlying mechanisms relating to the effect of exercise on melatonin and sleep (e.g. effects on core body temperature and additional biomarkers) were not specifically examined in this investigation. Despite these limitations, salivary melatonin concentrations increased from baseline to the end of the intervention, suggesting that the exercise intervention had a significant impact on melatonin production and its resulting effects on sleep quality.

\section{Conclusions}

Eight weeks of moderate-intensity aerobic exercise appears to increase onset nighttime salivary melatonin levels and may contribute to the improved self-reported sleep quality in previously sedentary adult men. Although literature is sparse and inconclusive, understanding melatonin's response to exercise is important in order to utilize best practices for sleep outcomes. This current study adds support to the understanding of melatonin's role during and after chronic exercise. The findings of this study suggest that chronic exercise may provide an effective approach for improving sleep due to the synchronization of melatonin with the circadian rhythm. Healthcare professionals may be able to use this information when discussing avenues to improve sleep in individuals who experience sleeping problems. This study does not suggest that exercise is the only mechanism to improve sleep, and recognizes that for certain individuals, pharmaceutical and medical interventions are necessary in order to appropriately treat certain sleep disorders. However, regular exercise may be a cost-effective avenue to improve sleep, either alone or in concert with other therapies. 


\section{Acknowledgements}

This work was supported by Elon University Faculty Research \& Development funds. The authors would like to thank Sarah Russell in the Department of Physical Therapy Education at Elon University for her assistance with data collection, as well as the 17 individuals who participated in this study. The authors have no potential conflicts of interest to disclose.

\section{Funding Source}

This work was supported by Elon University Faculty Research \& Development funds.

\section{Statement of Equal Authors' Contribution}

All persons who meet authorship criteria are listed as authors, and all authors certify that they have participated sufficiently in the work to take public responsibility for the content, including participation in the concept, design, analysis, writing, or revision of the manuscript.

\section{References}

1. Kelley GA, Kelley KS (2017) Exercise and sleep: a systematic review of previous meta-analyses. J Evid Based Med 10: $26-36$.

2. Kashefi Z, Mirzaei B, Shabani R (2014) The effects of eight weeks selected aerobic exercises on sleep quality of middle-aged non-athlete females. Iran Red Crescent Med J 16: 16408.

3. Montgomery P, Dennis J (2002) Physical exercise for sleep problems in adults aged $60+$. Cochrane Database Syst Rev 4: CD003404.

4. Baldursdottir B, Taehtinen RE, Sigfusdottir ID, Krettek A, Valdimarsdottir HB (2017) Impact of a physical activity intervention on adolescents' subjective sleep quality: a pilot study. Glob Health Promot 24: 14-22.

5. Kianian T, Navidia A, Aghamohamadi F, Saber S (2017) Comparing the effects of aerobic and anaerobic exercise on sleep quality among male nonathlete students. Nurs Midwifery Stud 6: 168-173.

6. Chen LJ, Fox KR, Ku PW, Chang YW (2016) Effects of aquatic exercise on sleep in older adults with mild sleep impairment: a randomized controlled trial. Int J Behav Med 23: $501-506$.

7. Kessler RC, Berglund PA, Coulouvrat C, Hajak G, Roth T, et al. (2011) Insomnia and the performance of US workers: results from the America insomnia survey. Sleep 34: 11611171.

8. Claustrat B, Brun J, Chazot G (2005) The basic physiology and pathophysiology of melatonin. Sleep Med Rev 9: 11-24.

9. Escames G, Ozturk G, Baño-Otálora B, Pozo MJ, Madrid JA, et al. (2012) Exercise and melatonin in humans: reciprocal benefits. J Pineal Res 52: 1-11.

10. Cajochen C, Kräuchi K, Wirz-Justice A (2003) Role of melatonin in the regulation of human circadian rhythms and sleep. J Neuroendocrinol 15: 432-437.

11. Marrin K, Drust B, Gregson W, Morris CJ, Chester N, et al. (2011) Diurnal variation in the salivary melatonin responses to exercise: relation to exercise-mediated tachycardia. Eur J Appl Physiol 111: 2707-2714.
12. Macchi MM, Bruce JN (2004) Human pineal physiology and functional significance of melatonin. Front Neuroendocrinol 25: 177-195.

13. Inoue S, Yorifuji T, Sugiyama M, Ohta T, Ishikawa-Takata $\mathrm{K}$, et al. (2013) Does habitual physical activity prevent insomnia? A cross-sectional and longitudinal study of elderly Japanese. J Aging Phys Act 21: 119-139.

14. Yang PY, Ho KH, Chen HC, Chien MY (2012) Exercise training improves sleep quality in middle-aged and older adults with sleep problems: a systematic review. J Physiother 58: 157-163.

15. Carlson LA, Pobocik KM, Lawrence MA, Brazeau DA, Koch AJ (2019) Influence of exercise time of day on salivary melatonin responses. Int J Sports Physiol Perform 14: 351-353.

16. Ammar A, Chtourou H, Souissi N (2017) Effect of Time-ofDay on Biochemical Markers in Response to Physical Exercise. J Strength Cond Res 31: 272-282.

17. Atkinson G, Drust B, Reilly T, Waterhouse J (2003) The relevance of melatonin to sports medicine and science. Sports Med 33: 809-831.

18. Buxton OM, Lee CW, L'Hermite-Balériaux M, Turek FW, Van Cauter E (2003) Exercise elicits phase shifts and acute alterations of melatonin that vary with circadian phase. Am J Physiol Regul Integr Comp Physiol 284: 714-724.

19. Thomas S, Reading J, Shephard RJ (1992) Revision of the physical activity readiness questionnaire (PAR-Q). Can $\mathrm{J}$ Sport Sci J 17: 338-345.

20. Backhaus J, Junghanns K, Broocks A, Riemann D, Hohagen F (2002) Test-retest reliability and validity of the Pittsburgh Sleep Quality Index in primary insomnia. J Psychosom Res 53: 737-740.

21. Matthew FJ (2005) Comparison of Bruce and Modified Bruce Treadmill Protocols. Journal of Cardiopulmonary Rehabilitation \& Prevention 25: 290.

22. Borg G (1998) Borg's Perceived Exertion and Pain Scales. Champaign, IL, US: Human Kinetics.

23. Pescatello LS, Arena R, Riebe D, Thompson PD (2014) ACSM's Guidelines for Exercise Testing and Prescription. (9 $9^{\text {th }}$ edn), Philadelphia, PA: Wolters Kluwer/Lippincott Williams \& Wilkins.

24. Shibata M, Tanida K, Shibata S, Kuzuhara K, Shimamoto $\mathrm{H}$, et al. (2011) Effects of daily physical activity level on circadian rhythm of oral body temperature and melatonin in children. J Strength Cond Res 25: S59.

25. Rhee EJ, Lee WY, Yoon KH, Yoo SJ, Lee IK, et al. (2010) A multicenter, randomized, placebo-controlled, double-blind phase II trial evaluating the optimal dose, efficacy and safety of LC 15-0444 in patients with type 2 diabetes. Diabetes Obes Metab 12: 1113-1119.

26. Cai ZY, Chen KWC, Wen HJ (2014) Effects of a groupbased step aerobics training on sleep quality and melatonin levels in sleep-impaired postmenopausal women. J Strength Cond Res 28: 2597-2603.

27. Carr DB, Reppert SM, Bullen B, Skrinar G, Beitins I, et al. (1981) Plasma melatonin increases during exercise in women. J Clin Endocrinol Metab 53: 224-225.

28. Theron JJ, Oosthuizen JM, Rautenbach MM (1984) Effect of physical exercise on plasma melatonin levels in normal volunteers. S Afr Med J 66: 838-841.

29. Monteleone P, Maj M, Fusco M, Orazzo C, Kemali D (1990) Physical exercise at night blunts the nocturnal increase of plasma melatonin levels in healthy humans. Life Sci 47 : 1989-1995. 